\title{
Sitzung vom 25. Juli 1904.
}

Vorsitzender: Hr. E. Buchner, Präsident.

Nachdem das Protocoll der letzten Sitzung genehmigt ist, ergreift der Vorsitzende das Wort zu folgendem Nachruf:

-Wiederum muss ich von einem Trauerfall Kenntniss gebev. Am 22. Juli starb zu Höchst am Main im Alter von nur 56 Jahren unser mehrmaliges Vurstandsmitglied, der Geh. Regierungsratb, Prof. Dr.

\section{AUgust LAUBENHEIMER.}

Als Privatdocent und Professor in Giessen hat sich der Verstorbene zuerst durch seine Betheiligung an der Erforschung der aromatischen Verbindungen, insbesondere der gechlorten Nitrobenzole, Nitroas iline und Nitrophenole, und durch Herausgabe eines trefflichen Lehı uaches *Grundzüge der organischen Chemie» bekannt gemacht. Dann in die Farbwerke vorm. Meister, Lucius \& Brüning in Höehst am Main berufen, hat er viele Jahre der Leitung dieses grossen Unternehmens gewidmet und es ausgezeichnet verstanden, wissenschaftlichen Geist mit industrieller Tbätigkeit zu vereinen. Wer mit $L$ a u benhei mer geschäftlich zu thun hatte, wird seine vornehme, liebenswürdige and sachliche Art bei der Leitung von Verhandlungen zu würdigen wissen. Nach meiner Erinnerung ist es nock kein Jahr, dass sich der Verstorbene von der Directorstelle zurückgezogen hat. Es entbehrt nicht der Tragik, dass ihm nur eine so kurze Ruhestandszeit vergönnt war.«

Die Versammelten erbeben sich zur Ehrung des Verstorbenen von ihren Sitzen.

Sodann begrüsst der Vorsitzende den auswärtigen Vicepräsidenten der Gesellschaft, Hru. Geheimrath Prof. Dr. Th. Curtius (Heidelberg), welcher im Laufe des wissenschaftlichen Theiles der Sitzung in zusammenfassender Weise einen Ueberblick seiner Untersuchungen - Ueber die besonderen Reactionen bei der Lmlagerung der Berichte d. D. chem. Gesellschaft. Jahrg. XXXVII. 
Säureazide giebt, sowie Hrn. Priratdocent Dr. F. W. Hinrichsen (Aachen).

Als ausserordentliche Mitglieder sind aufgenommen die HHrn.:

Stillman, Prof. Dr. Th. R., Hoboken;

Löwe, Dr. H., Freiburg;

Cavazzi, Prof. Dr. A., Bologua;

Hofmann, K., Heidelbery;

Suckert, Dr. F., Mälhansen;

Wolf, R., Charlottenburg;

Craveri, M., Berlin;
Alsleben, P., Berlin;

Sutter, Th., :

Weiss, V.,

Manasse, W., "

Stein, H.,

Späte, F., Dresden;

Siemens, F. G., Dreeden.

Als ausserordentliche Mitglieder werden vorgeschlagen die HHrn.: Meytr, Dr. Erwin, Warrington, England (durch J. F. Kronstein, Dr. A., Rheinbabnstr. 18, Karls- Holtz und ruhe R. Daum);

Lindenbayn, Dr. Hans, Niederstriegis, Sachsen

Köbler, Alfred, Moltkestr. 2, Jena

Oppe, Alfred, Sonnenbergstr. 3, Jena

(durch L. Wolff und

R. Marburg);

Eyde, S., Raadhusgaden 20, Christiania (durch R. Wolffenstein und H. Simonis);

Gyr. Dr. Joseph, Einsiedeln (durch A. Bistrzycki und A. (Gockel);

Stackelberg, Dipl.-Ing. Erast Baron von, Gut Lassinorm, Station Rakke, Russland, Estland (durch R. r. Walther nnd A. Lottermoser).

Für die Bibliothek sind als Geschenke eingegangen:

143. Bulietin of the United States Geological Survey, No. 208, 218-235, 227. Washington 1904 .

773. Sanmlung chemischer und chemisch-technischer Vorträge; herausgegeben von F. R. Ahrens. IX. Band, Heft 8, H. Ka ufmann: Ueber den Zusammenhang zwischen Farbe und Constitution bei chemi8chen Verbindungen. Stuttgart 1904.

833. Walcott, C. D. Twenty-fourth Annual Report of the United States Geological Surver, 1902-1903. Washington 1904.

841. Walcott, C. D. Professional Papers of the Department of the Interior (United States Geological Surrey) No. 11, 12, 16-20. Washington 1904.

844. Walcott. C. D. Mineral resoarces of the United States. U. S. Geological Surrey. Calendar Year 1902. Washington 1904. 
847. Bailey, W. S. The Menominee iron-bearing district of Michigan. (Monographs of the United States Geological Survey, Vol. 46.) Washington 1904.
Der Vorsitzende:
Der Schriftführer:
E. Bachaer.
i. F. A. Bannow.

\section{Nittheilnngen.}

\section{A. Werner und A. Grob: 9.10-Diphenyl-phenanthren, ein Product intramolekularer Umlagerungen.}

(Eingegangen am 30. Juni 1904; mitgeth. in der Sitzung von Hrn. O. Diels.)

Durch eine Reihe synthetischer Reactionen war es uns möglich über die Natur einer Anzahl hochmolekularer Kohlenwasserstoffe Klarheit zu gewinnen, und der dadurch ermöglichte Einblick in die Bildungoweise dieser Verbindungen stellt eine wesentliche Ergänzung unserer Kenntnisse intramolekularer Umlagerungen vom Typus der beim Pinakolylalkohol beobachteten dar. Wir haben uns im besonderen nit der Aufklärung der Constitution eines von $\mathrm{K}$ linger und Lonnes ${ }^{1}$ ) beschriebenen Kohlenwasserstoffes der empirischen Zusammensetzung $\mathrm{C}_{26} \mathrm{H}_{18}$, der durch Reduction von Diphenylendiphenylpinakolin entsteht, beschäftigt.

Als Ausgangsproduct zur Darstellung dient Dipbenylenphenylmethan (Phenylfuoren). Ueber die Constitution dieser Verbindung finden sich in der Literatur widerstreitende Ansichten. Hanriot und St. Pierre ${ }^{2}$ ), welche diesen Kohlenwasserstoff durch Erhitzen von Triphenylmethan mit Kalium auf $240^{\circ}$ erhielten, ertheilten ihm folgende Constitution: ${ }_{\mathrm{C}_{6}} \mathrm{C}_{5} \mathrm{H}_{5}>\mathrm{C}: \mathrm{C}_{6} \mathrm{H}_{4}$. Auch aus Triphenylchlormethan ${ }^{3}$ ) entsteht dieser Kohlenwasserstoff beim Erhitzen auf $250^{\circ}$, und ebenso aus Triphenylbrommethan $\%$. Die structurelle Auffassung von Hanriot und St. Pierre ist aber unhaltbar, wie aus der Gesammtheit der im Folgenden beschriebenen Thatsachen herrorgeht.

1) Diese Berichte 29, 2152 [1896].

2) Diese Berichte 22, Ref. 439, 660 [1889].

3) Hemilian, diese Berichte 7, 1208 [1874]; E. und O. Fischer, Ann. d. Chem. 194, 256 [1878].

*) H. Sehwarz, diese Berichte 14, 1522 [1881]. 